\title{
Enfoques y perspectivas contemporáneas del proyecto cosmopolita de los derechos humanos
}

José Lira Rosiles

\begin{abstract}
Esta investigación tiene como propósito realizar una cartografía de las corrientes y variantes principales de cosmopolitismo contemporáneo, explorando sus dimensiones y alcances. El cosmopolitismo es una corriente filosófica, jurídica y política muy diversa, siendo incorrecta su interpretación como un paradigma homogéneo que se decanta en la propuesta de un Estado de Estados, civitas maxima o Estado mundial. Esta consideración es importante sobre todo al evaluar la crítica anticosmopolita de pensadores como Carl Schmitt o Danilo Zolo. Tras esta exploración el artículo tiene como propósito resaltar que la categoría ciudadano del mundo (kosmopolites, Weltbürger) constituye el concepto central del cosmopolitismo contemporáneo de carácter federalista, el cual tiene como núcleo normativo y jurídico la doctrina de los derechos humanos.
\end{abstract}

El cosmopolitismo es una filosofía moral, jurídica y política cuyo pensamiento central es el desarrollo de un orden global justo y pacífico, planteando cuestiones acerca del ámbito de la justicia, la ley y el trato con aquellas personas que no son miembros de la familia, ciudad o Estado, con aquellos que son ajenos a las relaciones internas de la comunidad. Ya desde la antigüedad, el cosmopolitismo es una corriente filosófica que comprende una diversidad de enfoques y propuestas divergentes en relación con la naturaleza del orden global, así como las relaciones de los pueblos y los individuos a nivel mundial. Por otro lado, históricamente los actores centrales en las relaciones entre pueblos han sido los príncipes, soberanos o Estados, antes que los individuos. Observamos una característica esencial de los proyectos de paz pensados desde la modernidad temprana, básicamente desde el Abate de Saint-Pierre, Immanuel Kant hasta Hans Kelsen: la participación central de los Estados y potencias, antes que los individuos, pensados no sólo como ciudadanos estatales sino como ciudadanos del mundo. La política y el derecho internacional han sido ámbitos privilegiados para aquellos que detentan la autoridad y el poder. No obstante, una característica fundamental de los enfoques cosmopolitas contemporáneos es la centralidad del ciudadano del mundo en el proyecto de reforma democrática de las instituciones globales. Frente a la guerra, los adquiere un valor fundamental para la búsqueda de acuerdo 
y convivencia pacífica global, participación democrática y diálogo intercultural en un plexo de organismos y organizaciones trasnacionales e internacionales, cuyo actor privilegiado y sujeto de derecho global es el ciudadano del mundo.

\section{Fundamentos kantianos del cosmopolitismo contemporáneo}

El cosmopolitismo se define como una doctrina filosófica, jurídica y política para la paz, cuya negación de la guerra tendría su expresión más clara y sistemática en el pensamiento de Immanuel Kant. En su obra Idee zu einer allgemeinen Geschichte in weltbürgerlicher Absicht (1784), comprende el desarrollo histórico hacia un estado general cosmopolita y la asociación ciudadana completa de la especie humana como el fin supremo de la Naturaleza (Idee, AA VIII 28, 29). En el quinto principio de Idee, postula como el mayor problema para la especie humana, y cuya solución es forzada por la Naturaleza, la instauración (Erreichung) de una sociedad civil que administre universalmente el derecho. Esta sería una sociedad en la que la libertad bajo leyes externas se encontrara vinculada por una constitución civil (bürgerliche Verfassung) perfectamente justa. Las inclinaciones no pueden coexistir en un contexto de libertad salvaje, sino en un estado de coerción, donde sólo éstas pueden producir el mejor resultado. En el sexto principio, Kant afirma que este problema es el más difícil y el que más tardíamente será resuelto por la especie humana.

En el séptimo principio, el problema de la instauración de aquella constitución civil perfecta aparece como dependiente del problema de una reglamentación de las relaciones interestatales. En Idee, utiliza en sentido fuerte la analogía entre los dos estados de naturaleza: el primero, a nivel de relaciones interindividuales, que obliga a la instauración de una constitución civil; el segundo, a nivel de relaciones interestatales. Aquella insociable sociabilidad (ungesellige Geselligkeit) juega también un papel determinante en este segundo nivel donde, en razón de la incompatibilidad de las grandes sociedades y cuerpos políticos, en un inevitable antagonismo, predomina un estado de guerra. Para Kant, la guerra, como manifestación del ungesellige Geselligkeit a nivel de relaciones entre Estados, en el estado actual en el que se encuentra la cultura, constituye un medio indispensable para seguir haciéndola progresar, siendo que sólo después de haberse consumado sería 
provechosa una paz perpetua (immerwährender Friede), sólo posible en virtud de ella (MA, AA VIII 121). ${ }^{1}$ No obstante, tal situación conduce finalmente a los Estados a abandonar aquel estado sin ley (gesetzlosen Zustand) propio de los salvajes e ingresar en una federación de pueblos (Völkerbund, foedus amphictyonum). De tal modo, la seguridad y el derecho del Estado depende no de su propio poder, sino de un poder unificado (vereinigte Macht), de un estado cosmopolita de la seguridad pública estatal y de la decisión conforme a las leyes de la voluntad común.

En el octavo principio, Kant afirma, como corolario del anterior, que la historia de la especie humana puede considerarse, en conjunto, como la ejecución de un plan oculto de la Naturaleza (verborgenen Plans der Natur) para instaurar una constitución interior y exterior perfecta, como el único estado donde se pueden desarrollar en plenitud todas las disposiciones de la humanidad. Kant considera este desarrollo como un quiliasmo (Chiliasmus), cuyo advenimiento es contribuido por su propia idea, distando de ser quimérico o de ser una novela. En este desarrollo, piensa que la Ilustración, así como cierto interés del corazón por lo bueno, emerge poco a poco, llegando hasta los tronos y ejerciendo influencia sobre los principios de gobierno. Es esta lógica la que Kant describe en Das Ende aller Dinge (1794), como aquel continuo progresar y acercamiento al sumo bien (höchsten Gut, ED, AA VIII 335). Por tanto, la guerra se convertirá poco a poco en algo muy artificioso y dudoso, aunado a su rechazo en razón de las funestas consecuencias para el Estado con la deuda pública que acarrea, además de los perjuicios a la cultura y a la

\footnotetext{
${ }^{1}$ En Zum ewigen Frieden, Kant señala que por medio de la guerra la organización provisional de la naturaleza ha llevado a las personas a todas partes para poblarlas, incluso a las más inhóspitas (Frieden, AA VIII 363). En relación con el tema de la guerra en la filosofía de Kant, véase la obra Función y crítica de la guerra en la filosofía de Immanuel Kant, donde Santiago Oropeza pondera el análisis del problema de la guerra en la filosofía de la historia de Kant para enlazar los otros aspectos de su filosofía moral, tal como el proyecto de paz perpetua. La autora señala tres tesis de Kant, que en principio parecen discordantes, en torno a la guerra: 1) la guerra como origen del estado de derecho; 2) la guerra como mecanismo de la naturaleza (o la providencia) para instaurar un derecho internacional y; 3) la guerra como expresión de todos los males y la depravación de las costumbres. En este sentido, Santiago Oropeza señala la tensión en el pensamiento de Kant entre dos perspectivas: "por una parte, su concepción de que la guerra es un mecanismo dispuesto por la naturaleza para forzar al hombre al progreso moral y político y, por otra parte, su proyecto de paz y el mandato de la razón práctica que prohíbe la guerra" (Santiago, 2004: 15). Santiago Oropeza argumenta que no hay discrepancia entre ambas perspectivas en la filosofía de Kant.
} 
libertad (MA, AA VIII 121). Estas consecuencias son importantes dada la interrelación comercial de los Estados, que es ponderada hacia la parte final de Idee, por lo que éstos se constituyen provisionalmente como árbitros, aun sin tener la autoridad legal para ello, pero preparándose así para integrar un gran cuerpo estatal (großen Staatskörper). Tal cuerpo político se presenta hasta ahora en la forma de un tosco esbozo, pero que acabará por constituirse, de acuerdo con el propósito de la naturaleza, en aquel estado cosmopolita universal (allgemeiner weltbürgerlicher Zustand) en el que se desarrollaran plenamente todas las disposiciones de la especie humana.

El tema del estado universal cosmopolita es retomado por Kant en su obra Zum ewigen Frieden (1795), donde planteó la superación del estado de naturaleza que subsiste entre los Estados para instaurar un estado civil internacional a través de una unión de Estados (Staatenverein), una federación mundial cosmopolita, compuesta por Estados libres y fundada en un derecho cosmopolita (Weltbürgerrecht, ius cosmopoliticum) (Frieden, AA VIII 349).

Tal condición debe ser instaurada, ya que la paz no es un estado natural (Naturzustand, status naturalis), sino que lo es el estado de guerra (Zustand des Krieges). La guerra, que se había manifestado en Idee y Mutmaßlicher Anfang der Menschengeschichte como una expresión del ungesellige Geselligkeit, acompañando el progreso y el avance de la cultura, aparece en Zum ewigen Frieden como el triste medio necesario por el cual los Estados imponen su derecho en el estado de naturaleza, en razón de la no existencia de un tribunal de justicia que pueda juzgar con la fuerza de la ley, la decisión del lado en qué está el derecho, depende en tal condición de la inclinación de cada Estado.

Para Kant, la razón condena la guerra como una vía jurídica, convirtiendo el estado de paz (Friedenzustand) en un deber inmediato, fundado por un pacto entre los pueblos, conseguido a través de una federación de la paz (Friedenbund, foedus pacificum), que buscaría finalizar para siempre todas las guerras. En este sentido, distingue entre una foedus pacificum y un pactum pacis (Friedensvertrag, tratado de paz), ya que éste último sólo busca finalizar una guerra, no obstante, el estado de guerra no es anulado por él. La foedus 
pacificum no tiene como propósito la adquisición de un poder de corte estatal, sino el asegurar la libertad de los Estados federados, sin que estos deban someterse a leyes públicas y su coacción, tal como ocurre a nivel de los individuos en el nivel interno. Kant afirma que, según la razón, no existe otra manera de salir del estado de naturaleza en las relaciones entre Estados, que de la mis ma manera como los individuos crean leyes públicas coactivas, esto es, constituyendo un estado de pueblos (Völkerstaat, civitas gentium) que comprenda a todos los pueblos de la tierra. No obstante, introduce una tesis central: los pueblos, de acuerdo con su idea del Völkerbund, no quieren esto en absoluto, rechazando in hypothesi lo que es correcto in thesi, optando por el sucedáneo negativo (negative Surrogat) de una federación, y no por la idea positiva de una república mundial (Weltrepublik). ${ }^{2}$

\section{Problemas y tensiones en el proyecto jurídico-político cosmopolita}

La tensión ínsita en la filosofía política internacional de Kant, ha dado pauta al surgimiento de interpretaciones fuertes que respaldan la tesis de un Estado o república mundial, así como interpretaciones débiles que apoyan la tesis de un esquema federativo global, la

\footnotetext{
${ }^{2}$ Considero que, aunque Kant concibe al Estado mundial como una idea de la razón que debe admitirse como posible en la práctica, podemos encontrar en su filosofía, razones y argumentos consistentes en contra de la idea de una instauración inmediata, violenta y no consensuada del mismo. Podemos construir, frente a los teóricos kantianos que proponen la tesis del Estado mundial, tal como Thomas Pogge, Louis-Philippe Hodgson, Thomas Carson o Pauline Kleingeld, un argumento kantiano contra la idea del Estado mundial basado en su concepción del Estado como persona moral (moralische Person) y el argumento de la autonomía de los ciudadanos que conforman el mismo. En el segundo y quinto artículos preliminares hacia la paz perpetua, encontramos sólidos fundamentos que contrastan con la idea de una formación inmediata del Estado mundial. En el segundo artículo preliminar, el Estado no aparece como un patrimonium, como una propiedad, sino una comunidad de personas sobre la que ningún otro, más que ella misma, puede mandar y disponer (Frieden, AA VIII 344). La anexión de un Estado como un injerto en otro Estado, significaría anular la existencia del mismo en tanto persona moral, haciendo de él una cosa (eine Sache). ${ }^{2}$ Ello, además, implicaría contradecir la idea del contrato originario, sin el cual no es posible pensar un derecho sobre un pueblo. Por otro lado, en el quinto artículo preliminar, Kant establece que ningún Estado debe inmiscuirse por la fuerza en la constitución y gobierno de otro Estado. La intromisión de poderes externos (äußerer Mächte) constituiría una violación de los derechos de un pueblo independiente, poniendo en peligro la autonomía de todos los Estados. Sobre la interpretación de la inclinación de Kant por la tesis de la Federación de Estados y el rechazo de un Estado de Estados véase: Carson, 1998: 176; Habermas, 2014: 332; Held, 1997: 274; Höffe, 2006: 189; Leyva, 2009: 290; Mertens, 1996: 330; 2005: 87; Mikalsen, 2011: 306, Nussbaum, 2007: 234; Pogge, 2009: 144; Santiago, 2004: 147; 2008: 56, 170).
} 
inclinación por la tesis fuerte o débil tiene consecuencias importantes en la visión cosmopolita sobre la paz y la guerra. Existe una prolífica discusión en torno a si el proyecto de cosmopolitismo político y jurídico implica la constitución de un Estado mundial (Weltstaat), Estado de pueblos (Völkerstaat), Estado universal o civitas maxima. La crítica anticosmopolita de Carl Schmitt (1932) relaciona el proyecto de pacificación jurídica entre naciones con la constitución de un Estado mundial (Weltstaat), destruyendo la visión del pluralismo de los Estados como la esencia del concepto de lo político. La formación de un Estado mundial conllevaría a la muerte de lo político y del Estado soberano en un estadio de despolitización definitiva y una homogeneización cultural y jurídica. En la línea crítica de Carl Schmitt, Danilo Zolo (2000) argumenta que en la propuesta de los globalistas occidentales se configura una moderna forma de Cosmópolis, en la que las relaciones entre Estados se someten al control y poder de intervención de un gobierno mundial. La acusación del anticosmopolitismo es clara: los proyectos de cosmopolitismo político y jurídico implican la instauración de una determinada clase de república o gobierno mundial.

En términos generales, Zolo (2000: 13) considera que, en el plano político, tras la caída del imperio soviético y el fin del bipolarismo se ha reforzado una perspectiva globalista (cuyos representantes son llamados irónicamente por Zolo, siguiendo a Hedly Bull, como globalistas occidentales [Western globalist]), junto con la consolidación, a partir de la guerra del Golfo Pérsico, de la praxis del intervencionismo humanitario y la instauración de instituciones propias de una jurisdicción penal internacional, como el Tribunal Penal Internacional. Los globalistas occidentales han predicho una fuerte concentración del poder internacional, en razón del desfase entre los procesos de la globalización y la capacidad de la soberanía de los Estados nacionales para afrontar los problemas de dimensión global. Los globalistas consideran que resulta anacrónico considerar que la garantía del orden mundial y el mantenimiento de la paz puedan seguir basándose en el modelo westfaliano, surgido en el siglo XVII, basado en el equilibrio entre potencias europeas. De tal modo, proponen una reforma de las instituciones internacionales, en particular de Naciones Unidas, para transformarlas en auténticos centros de poder 
supranacional que estén por encima de la soberanía de los Estados nacionales, es decir, los globalistas afirman la necesidad de una nueva jerarquía de poder internacional formalmente establecido y legitimado. Para Zolo, los globalistas abogan por una forma moderna de Cosmópolis, en las que las relaciones entre Estados, y entre los Estados y los individuos, estén sometidas al control y poder de intervención de un gobierno mundial. ${ }^{3}$ Zolo (2000: 140) denomina como cosmopolitismo legal a la doctrina en la que confluyen cuatro tesis normativas: primera, la primacía del derecho internacional y la progresiva reducción de soberanía de los Estados; segunda, el centralismo jurisdiccional, es decir, el proceso de desarrollo de normas y organismos centralizados para la aplicación coercitiva de la ley; ${ }^{4}$ tercera, el pacifismo legal, que intenta imponer límites a la guerra a partir de la aplicación efectiva del derecho internacional y; cuarta, el constitucionalismo global, como una tesis vinculada a la teoría de los derechos humanos, que "subraya la capacidad de las instituciones supranacionales centralizadas para salvaguardar las libertades individuales fundamentales de los individuos que los propios Estados han sido incapaces de proteger" (Zolo, 2000: 141). Esta última tesis comprende una serie de supuestos, por ejemplo, que los derechos humanos y su universalidad pueden ser defendidos racionalmente; que los derechos humanos son derechos en el pleno sentido de la palabra, que pueden ser definidos con precisión o que son derechos positivos, vinculantes jurídicamente; finalmente, que los derechos humanos no pueden ser plenamente garantizados por los Estados nación, ni siquiera por los más democráticos y liberales. ${ }^{5}$ Para Zolo, la doctrina de los derechos

\footnotetext{
${ }^{3}$ Los iusglobalistas proponen una reforma de los organismos internacionales que los transforme en auténticos poderes supranacionales, capaces de superponerse a la soberanía de los Estados nacionales y de limitar drásticamente su domestic jurisdiction" (Zolo, 2005: 199).

${ }^{4}$ En el caso del modelo propuesto por Hans Kelsen, en una etapa inicial la aplicación coercitiva de la ley tiene como característica la descentralización. No obstante, para Kelsen, el desarrollo gradual de un Estado Federal Mundial tiene, en efecto, la característica esencial de ser un orden centralizado. No obstante, no puede suscribirse a Kelsen la idea de que tal Estado Federal Mundial se constituya en un Estado Mundial Unitario. (Véase, Kelsen, 1986: 181; 2008: 47, 122; Leben, 1998: 289).

${ }^{5}$ Luigi Ferrajoli (2008: 71) responde a este argumento de Zolo, afirmando que descansa en una confusión entre derechos y garantías. Las garantías constituyen obligaciones correspondientes a derechos subjetivos, existiendo garantías primarias, referidas al establecimiento de tales derechos en normas, y garantías secundarias, referidas al establecimiento de órganos judiciales que sancionen violaciones a los mismos. La de
} 
humanos presentaun sesgo cosmopolita, ya que a diferencia del Jaus Publicum Europaeum, considera como sujetos del orden jurídico internacional no sólo a los Estados sino también a los individuos. La doctrina de los derechos humanos, comenta Zolo, tiende a construir una ciudadanía cosmopolita, que comprende una ciudadanía y un sistema jurídico sin fronteras, basados en los derechos fundamentales de todos los seres humanos en cuando seres racionales y personas. Es así como para Zolo, la doctrina de los derechos humanos remite, a modo kantiano y kelseniano, a la idea de una comunidad universal, de una civitas maxima. La protección internacional de los derechos humanos conlleva a la tesis de un constitucionalismo mundial, lo que implica, afirma Zolo, que el modelo europeo de estado de derecho se adopte universalmente, con lo que se busca constituir una Cosmópolis de derecho basada en los valores políticos occidentales. Zolo (2005:198) encuentra el fundamento del proyecto del globalismo jurídico en la idea kantiana de la unidad del género humano y la idea de una globalización del derecho en la forma de una legislación universal, lo cual implicaría una homologación gradual de las costumbres y ordenamientos normativos nacionales, así como de las diferencias políticas y culturales.

\section{Hacia una cartografía del cosmopolitismo jurídico y político contemporáneo}

Uno de los aspectos fundamentales para evaluar la crítica al cosmopolitismo contemporáneo consiste en realizar en primera instanciar una cartografía de las propuestas de diversos autores cosmopolitas. Es común encontrar la consideración del proyecto cosmopolita como un paradigma homogéneo, cuya naturaleza se inscribe en la instauración de un Estado de Estados, civitas maxima o Estado mundial, implicando un proceso de homogeneización política, económica, jurídica y cultural que finalmente conforma la política global del imperialismo occidental. No negamos la pertinencia teórica y práctica de tales críticas, en razón del desarrollo histórico del sistema internacional y su actual

existencia de las primeras y la ausencia de las segundas no se puede afirmar la inexistencia de los derechos fundamentales. 
situación. No obstante, el proyecto cosmopolita contemporáneo dista de suscribir tal visión, constituyéndose a su vez como un paradigma crítico a tales tesis. En este sentido, y como ocurre con las más destacadas corrientes de pensamiento, es fácil elaborar esquematizaciones sobre-simplificadoras que conducen a una comprensión incorrecta del mismo. En esta cartografía distinguiremos analíticamente entre aquellos teóricos próximos a la idea de una república mundial, Estado de Estados o Estado universal (a) y aquellos próximos a la tesis de un sistema global democrático (b), en la que los Estados conservan un estatus político y legal independiente, sin someterse a un Estado en analogía con el nivel interno. La tesis de un Estado universal se fundamenta en la constitución de una entidad supraestatal con plena capacidad coactiva, en la que los Estados son integrados, y nunca entendidos como unidades independientes. La tesis de un sistema internacional, por el contrario, comprende a los Estados como unidades independientes y necesarias, que han de participar democráticamente en entidades y organismos de cooperación y gobernanza a nivel trasnacional y supranacional. ${ }^{6}$ Ahora bien, (a) y (b) deben comprenderse en sentido graduado, ya que podemos encontrar versiones fuertes y débiles en ambos grupos.

\section{(a) Tesis de la República universal o Estado mundial}

La tesis fuerte a favor de la constitución de un Estado mundial se deriva de la crítica de la elección de Kant por la idea de una federación de pueblos, lo cual se ha visto por autores neokantianos como un movimiento que no es coherente con su sistema filosófico. De acuerdo con estos autores, el Estado mundial o República mundial puede encontrar

\footnotetext{
${ }^{6}$ Para esta división me baso fundamentalmente en Gustav Radbruch (2007), quien entiende al grupo (a) como la concepción individualista del Estado, en la que el Estado singular está destinada a disolverse en un futuro Estado universal y; al grupo (b) como la concepción de transpersonalismo cultural, el cual defiende el estatus del Estado moderno como sujeto del derecho internacional, negando su disolución en un Estado universal, ponderando en cambio la centralidad de la Sociedad de Naciones. Radbruch identifica al transpersonalismo cultural como la tendencia evolutiva del derecho internacional y la concepción de los Estados como miembros de una comunidad jurídica. Podemos encontrar un división analítica similar en Mikalsen (2011), quien identifica dos núcleos teóricos en los proyectos de establecimiento de un sistema de derecho internacional: primero, las propuestas de modelos intergubernamentales mínimos (minimal intergovernmental models) y; segundo, las propuestas que abogan por una república mundial (world republic). Mikelsen divide al segundo grupo entre los proponentes de un Estado de Estados (state of states) y aquellos que abogan por una democracia cosmopolita.
} 
justificación y fundamentos conceptuales sólidos partiendo de las premisas mismas establecidas por Kant. ${ }^{7}$ Encontramos, por un lado, aquellos teóricos que construyen una justificación kantiana del Estado mundial, cuya característica principal es que tal institución posee un carácter coercitivo, pudiendo obligar o forzar a los Estados reticentes a abandonar el estado de naturaleza internacional para ingresar a la condición civil global (a.1). Estos teóricos revitalizan la analogía entre los dos Estados de naturaleza, rechazada finalmente por Kant, proponiendo que así como los individuos pueden ser obligados a ingresar a una condición civil, un Estado mundial también puede obligar o forzar a los Estados individuales a salir del estado de anarquía internacional. El Estado mundial es pensado como un Estado mundial mínimo o ultra-mínimo, restringido y limitado de modo que no puede intervenir en los asuntos domésticos de los Estados, así como tampoco constituirse como una tiranía o despotismo global. En este grupo podemos clasificar a (a.1.1) Thomas Carson (1998) y su tesis de un gobierno mundial 'ultra-mínimo' ('ultra-minimal' world government $)^{8} 8$ y a (a.1.2) Louis-Philippe Hodgson (2012) y su tesis de un Estado mundial, de carácter federal, como garante global del derecho a la libertad (right to freedom). ${ }^{9}$ Tanto Carson, como Hodgson, consideran que los Estados individuales pueden ser forzados a ingresar al Estado mundial. ${ }^{10}$

\footnotetext{
${ }^{7}$ Para una interpretación que sostiene claramente esta postura, véase Höffe, 2006: 193.

${ }^{8}$ El 'ultra-minimal' world government propuesto por Carson, se caracterizaría por una constitución pública que prohíba el uso de la fuerza de una nación contra otra; un cuerpo judicial facultado para determinar las violaciones a tal constitución y ordenar el uso de la fuerza; así como un ejército o policía internacional para ejecutar las decisiones del órgano judicial (véase, Carson, 1998: 182).

${ }^{9}$ Hodgson (2010: 791; 2012: 103) considera a este derecho como fundamental en la filosofía de Kant, concerniente exclusivamente al ámbito de la libertad externa. Este derecho puede ser justificablemente restringido sólo en razón de la libertad misma, en el sentido de dificultar los obstáculos a la libertad (hindering of a hindrance to freedom). Tal derecho sólo podría realizarse en la condición civil, en un Estado de derecho que garantice y haga a los derechos conclusivos (Hodgson, 2010: 815). En este sentido: "To make rights conclusive at the international level, a state would have to make itself into a world state" (Hodgson, 2012: 117). Para un análisis del right to freedom en el contexto de una interpretación a favor de la liga de Estados, y no del Estado mundial, véase Mikalsen, 2011: 295.

10 "The right to freedom sets extremely high demands on what can count as a justified use of force, be it against states or against individuals" (Hodgson, 2012: 117). Para Carson, (1998: 183): "Kant fails to fully grasp the logic of his own argument", ya que así como los Estados poseen un poder coercitivo contra los Estados, un Estado internacional debería tener poderes coercitivos sobre los Estados individuales. Esta interpretación es rechazada, sobre principios kantianos, por Pauline Kleingeld (2004: 308), ya que un Estado de Estados que pudiera obligar a otros Estados a ingresar a aquella condición civil global podría violar la
} 
Ahora bien, encontramos otro grupo de teóricos kantianos (a.2) ${ }^{11}$ que construyen un argumento a favor de la tesis del Estado mundial, no obstante, niegan, revitalizando las preocupaciones y argumentos de Kant sobre el peligro de un despotismo global, la pertinencia de la utilización de la fuerza para obligar a otros Estados a ingresar al mismo. Estos pensadores reafirman la comprensión de Kant del Estado mundial como una idea de la razón que, no obstante, no ha de realizarse inmediatamente a través del uso de la fuerza, sino mediatamente a través de un proceso acentuadamente federativo que constituya un gobierno mundial en primera instancia soft, dentro de un proceso de cosmopolitismo graduado.

En este grupo clasificamos las siguientes propuestas: (a.2.1) el proyecto de una Liga Permanente para el Establecimiento de la Paz propuesto por Hans Kelsen, en su obra La paz por medio del derecho, cuyo organismo central es un Tribunal Internacional (1986: 182, 198; 2008: 47, 122); ${ }^{12}$ (a.2.2) el Sistema Internacional Democrático, pensado por Norberto Bobbio (2001: 198). Tal sistema sería instaurado con mediante el tránsito del estado agonístico al estado pacífico a nivel global, ello a través de no sólo de un pactum societatis sino de un pactum subiectionis que establezca un poder común fuerte, con el monopolio legítimo de la fuerza, en analogía con el establecimiento del poder civil a interno; ${ }^{13}$ (a.2.3) la República mundial (Weltrepublik) subsidiaria y federal, propuesta por Otfried Höffe como en un modelo mixto en el que los actores centrales son los ciudadanos

autonomía, autodeterminación y autolegislación de los ciudadanos del mismo. En este mismo sentido, Nussbaum (2007: 234, 256-58) rechaza la tesis fuerte de la coerción, aunque con el componente del acento en la categoría de soberanía.

${ }^{11}$ Mikalsen (2011: 292) identifica a los grupos (a.1) y (a.2) respectivamente como la crítica estandar y la interpretación del modelo por etapas. La crítica estandar pondera la analogía entre los dos Estados de naturaleza, así como la tesis fuerte de una República mundial. La interpretación del modelo por etapas, en donde Mikalsen identifica la visión de Kleingeld, propone que la liga de Estados no es el diseño institucional final para establecer relaciones internacionales justas, sino un primer paso que debe ser sustituido por un Estado de Estados cuando las condiciones sean las adecuadas.

${ }^{12}$ Para Kelsen, los Estados se adhieren voluntariamente a la Liga, si esta ha de tener un carácter democrático basado en la libertad e igualdad de los Estados miembros. El establecimiento de un poder ejecutivo centralizado constituye el problema más difícil de la organización internacional, pudiendo ser alcanzado sólo como uno de los últimos pasos, tras la consolidación del tribunal internacional.

${ }^{13}$ Zolo (2000: 61) considera que la propuesta de Bobbio de un tercero super partes, constituido a nivel internacional a través de un pactum subiectionis, significa establecer un super Estado o un Estado mundial, que posea el monopolio de la fuerza internacional. 
del mundo y los Estados, en una fase inicial soft o de cosmopolitismo graduado, ${ }^{14}$ (a.2.4) la propuesta de Pauline Kleingeld (2004: 318) de la constitución de un Estado de Estados, no de modo inmediato, a través de la fuerza, sino de modo mediato a través de una federación de Estados de carácter voluntario; (a.2.5) Thomas Pogge (2009: 151), en consonancia con Kleingeld, defiende la interpretación del proyecto de Kant como la propuesta de una República mundial, como aquella que puede posibilitar una condición jurídica plena a nivel global. Pogge crítica la oposición binaria en la teoría política de Kant entre un estado de naturaleza absoluto y una condición jurídica plena, ya que es posible sostener la idea de una condición semi-jurídica. Aquella oposición binaria no le permitió a Kant concebir un esquema multinivel en el que la autoridad política se encuentra verticalmente dispersa. Pogge piensa en este esquema como un gobierno mundial con agencias centrales encargadas de cumplir funciones legislativas, ejecutivas y judiciales, en un sentido cercano a la propuesta de la Ética discursiva (b.2).

\section{(b) Tesis de la Federación de Estados}

Los teóricos neokantianos proponentes de una reforma del sistema global en sentido democrático ponderan la centralidad del Estado entendido como un miembro independiente de la comunidad jurídica global. Esta perspectiva niega la pertinencia teórica y práctica, además de la deseabilidad, de la tesis de un Estado mundial $^{15}$ En su lugar, se pondera la necesidad de un sistema de gobierno o gobernanza global, entendido como un sistema que comprende instituciones y organizaciones civiles guiados dentro del marco del derecho legal internacional. En este grupo podemos clasificar las siguientes propuestas: The Law of peoples (b.1), ${ }^{16}$ donde John Rawls, desde el enfoque de la justicia como equidad (justice

\footnotetext{
${ }^{14}$ Véase Höffe (1999; 2004: 183; 2000: 247).

${ }^{15}$ Para un argumento claro de la no deseabilidad de un Estado mundial, véase Nussbaum (2007: 309), así como Held (1997: 275).

${ }^{16}$ Martha Nussbaum (2007: 229) considera The Law of Peoples como un ejemplo de la estrategia teórica en el ámbito de la justicia global de un contrato en dos fases: a nivel interno y a nivel externo, entre pueblos. Nussbaum defiende que esta obra de Rawls no puede ofrecer una teoría adecuada de la justicia global, ya que tampoco su proyecto fue pensar a ésta en general, sino que su objetivo sería describir la política exterior correcta para una sociedad liberal decente, así como las cuestiones relativas a la guerra y la paz.
} 
asfairness), extiende la idea general del contrato social a una sociedad de pueblos, compuesta por los pueblos democráticos liberales y por los "pueblos jerárquicos decentes". ${ }^{17}$ Rawls (1999: 36) sigue la idea expuesta por Immanuel Kant en Zum ewigen Frieden de una foedus pacificum (confederación pacífica de Estados), negando la tesis del Estado mundial (world-state), al que entiende como un régimen político unificado (unified political regime) con poderes legales ejercido normalmente por gobiernos centrales. Tal Estado mundial, piensa Rawls siguiendo la crítica de Kant a la monarquía universal, podría constituirse como un despotismo global (global despotism); ${ }^{18}$ (b.2) el sistema multinivel de Jürgen Habermas, quien rechaza explícitamente el telos de una república mundial. La Ética discursiva propone una federación con instituciones comunes (Föderation mit gemeinsamen Institutionen), la cual posee funciones estatales bajo la forma de una regulación jurídica, en el marco de una constitucionalización del derecho internacional, de los intercambios y el control de su cumplimiento en el ámbito de una política interior mundial (Weltinnenpolitik). El corolario de tal proyecto, es la participación democrática de una ciudadanía mundial en una sociedad civil y una opinión pública globales ${ }^{19}$; la idea una comunidad democrática cosmopolita, de carácter federal y voluntaria, propuesta por David Held (1997), apuntalada por un derecho democrático cosmopolita, ${ }^{20}$ en claro rechazo a la tesis del Estado mundial; (b.4) el sistema de gobierno global tenue y descentralizado (thin and decentralized) desarrollado por el enfoque de las capacidades, en claro rechazo del

\footnotetext{
${ }^{17}$ Rawls pondera la categoría de pueblos (peoples) y no de Estado como el actor central de la sociedad internacional, fundamentalmente porque los pueblos son capaces de tener un cierto carácter moral, ello derivado de su carácter racional y razonable. La categoría de Estado, en cambio, se define por una soberanía y un ámbito de autonomía que le permite guiarse por sus intereses racionales y prudenciales, esto es, por la razón de Estado (reason of state). Para Rawls (1999: 26), esta autonomía es nociva. (Véase también, Nussbaum, 2007: 244).

${ }^{18}$ Kleingeld (2004: 304) considera que en The Law of Peoples, la apelación de Rawls a las razones afirmadas por Kant para rechazar el ideal de un gobierno mundial, sirve como un atajo teórico que le permite evadir la discusión sobre la conveniencia de una Federación mundial de Estados. Ante todo porque: "Kant's objection to the formation of a universal monarchy does not imply the rejection of a federal state of states" (Ibid.: 313). Kleingeld (2004: 319) considera que el argumento general de Rawls contra la idea de un Estado de Estados no puede justificarse apelando a Kant.

${ }^{19}$ Véase Habermas (1993, 1995, 1998, 1999, 2000, 2014, 2008a, 2008 b).

${ }^{20}$ La propuesta Held (1997: 272) de un derecho democrático cosmopolita se basa en la distinción del öffentliches Recht establecida por Kant en Zum ewigen Frieden, por lo que se distingue del derecho estatal y del derecho internacional.
} 
Estado mundial $^{21}$; (b.5) el sistema de gobierno global de Mary Kaldor (2003, 2005), quien niega la tesis del Estado mundial, ya que aun si fuera constituido democráticamente: "would be totalitarian" (2003: 591). Kaldor propone, en cambio, como un modelo adecuado al proceso actual de globalización, la constitución de un sistema de gobierno global. ${ }^{22}$ Para Kaldor, tal modelo se constituye a través de un proceso de negociaciones y pactos entre instituciones y organizaciones estatales y regionales, así como a la sociedad civil global, superando con ello el horizonte del Estado centralizado y beligerante; (b.6) la Liga de Estados de Kjartan Mikalsen (2011), al interpretar, contra (a.1) y (a.2), a la liga voluntaria como la idea racional en el pensamiento de Kant, mientras que el Estado de Estados aparece en conflicto con el derecho y la justicia. ${ }^{23}$ La Liga de Estados se constituiría como una autoridad pública internacional con los poderes judiciales necesarios como el marco institucional constitutivo de una condición civil internacional; finalmente, (b.7) la propuesta del constitucionalismo mundial de Luigi Ferrajoli (2001, 2004a, 2004b), quien piensa que éste no requiere la institución de una forma de Estado a nivel supranacional, una especie de super-Estado mundial formado incluso a través de la democratización de Naciones Unidas. Ferrajoli cree que tal proyecto requiere de la introducción de instituciones y funciones de garantías no sujetas a la aprobación o anulación por ninguna mayoría o poder, lo que garantiza la consideración axiológica de los derechos fundamentales como la ley del más débil. ${ }^{24}$

\footnotetext{
${ }^{21}$ Véase Nussbaum (2007: 311), así como el capítulo I de esta investigación, donde se desarrolla en extenso la propuesta cosmopolita del enfoque de las capacidades.

22 "It is not a single world state, but a system in which states are increasingly hemmed in by a set of agreements, treaties and rules of transnational character" (Kaldor, 2003: 590). Tal sistema es entendido como: "un marco de normas que implican competencias que se solapan entre organizaciones internacionales, gobierno local, regional y Estados" (Kaldor, 2005: 146).

${ }_{23}$ Para Mikelsen, la tesis de un Estado de Estados es problemática en razón de que las fuertes asimetrías de poder determinarían que los Estados más fuertes establecieran los términos de la cooperación; así como la implicación del uso de la guerra para obligar a otros Estados a salir de estado de naturaleza internacional.

${ }^{24}$ De acuerdo con Ferrajoli, las instituciones de garantía son aquellas cuyas funciones están vinculadas a la aplicación de la ley y la tutela de los derechos humanos y la paz, es decir, sus funciones se encuentran comprendidas dentro de la esfera de lo indecidible, dentro de la esfera de los derechos fundamentales. De tal modo, las instituciones de garantía se distinguen de las instituciones de gobierno, cuya función por excelencia es la política y la administración, dentro de la esfera de lo decidible. De tal modo, primero, deben constituirse instituciones de garantía primaria, como las garantías jurídicas de paz y seguridad y las garantías jurídicas de derechos fundamentales que contemplen los derechos sociales, segundo, instituciones de garantía secundaria,
} 


\begin{tabular}{|c|c|c|}
\hline Estado mundial o República mundial (a) & & Federación de Estados (b) \\
\hline \multicolumn{3}{|l|}{ Tesis Fuerte (a.1) } \\
\hline (a.1.1) Carson: Gobierno Mundial ultra-mínimo & (b.1) & Rawls: Federación de pueblos. \\
\hline (a.1.2) Hodgson: Estado Mundial Federal & (b.2) & Habermas: Sistema Global Multinivel \\
\hline Tesis débil (a.2) & (b.3) & Held: Comunidad Democrática cosmopolita \\
\hline (a.2.1) Kelsen: Estado Federal Mundial gradual & $\begin{array}{c}\text { (b.4) } \\
\text { Descentralizado. }\end{array}$ & Nussbaum: Sistema de Gobierno Global Tenue y \\
\hline (a.2.2) Bobbio: Sistema Internacional Democrático & (b.5) & Kaldor: Sistema de Gobierno Global. \\
\hline (a.2.3) Höffe: República mundial soft. & (b.6) & Mikalsen: Liga voluntaria de Estados \\
\hline (a.2.4) Kleingeld: República mundial gradual. & (b.7) & Ferrajoli: Constitucionalismo mundial. \\
\hline (a.2.5) Pogge: República mundial multinivel & & \\
\hline
\end{tabular}

Cuadro 1. Espectro de propuestas cosmopolitas contemporáneas en torno al sistema de gobierno mundial.

El cosmopolitismo jurídico-político contemporáneo conforma un amplio espectro de propuestas graduadas derivadas de la interpretación conceptual del proyecto kantiano, así como su reformulación posible en la actual situación internacional. El concepto de ciudadanía mundial adquiere caracteres peculiares en tanto nos coloquemos en (a) o (b), ya que por ejemplo en (a) observamos la construcción de conceptualizaciones que tienden a minimizar o desdibujar tal participación, como es el caso de (a.1.1 y a.1.2); y en (b) el concepto de ciudadanía mundial y una sociedad civil y opinión públicas globales poseen una potencia mayor, al acentuar el carácter democrático, graduado y más horizontal en un proceso de gobernanza global. Observamos nítidamente cómo el cosmopolitismo esto es, de jurisdicciones con la capacidad de sancionar las violaciones a los derechos fundamentales, tal como la Corte Penal Internacional. 
contemporáneo dista absolutamente de ser una corriente homogénea, por lo que las críticas al mismo deben incluir matices importantes, no pudiendo suscribir al cosmopolitismo, en general, el propósito de constituir un Estado mundial o República mundial de carácter despótico, autoritario u homogeneizador, tal como encontramos en la crítica de Carl Schmitt o de Danilo Zolo. ${ }^{25}$

Ahora bien, tanto en el grupo de propuestas (a) como en (b) encontramos denominadores comunes: (ab.1) La tesis de un sistema de gobierno mundial de naturaleza federal, emprendido a través de un proceso global de institucionalización cuyo fundamento en el derecho internacional, así como la necesaria participación de una ciudadanía mundial como en el componente indispensable de tal proceso, con el matiz interpretativo sobre tal ciudadanía mencionado anteriormente; (ab.2) El cosmopolitismo contemporáneo tiene como denominador común la idea radical a la categoría de soberanía comprendida como potestas legibus soluta ac superiorem non recognoscens. Es importante señalar que éste no sostiene la idea de la desaparición del Estado: el Estado es conceptualizado como miembro de una comunidad jurídica como sujeto de derecho obligado. (ab.3) Es interesante notar que ambas corrientes de cosmopolitismo incluyen la posibilidad de intervenciones armadas con el fin de proteger los derechos humanos, de garantizar la aplicación de la ley (law enforcement) a nivel global, aunque cabe señalar que se acentúa la importancia de la existencia de una legislación humanitaria global clara, así como las instituciones y procesos adecuados para que tal intervención no se realice en función de razones de Estado sino en el marco de un orden legal internacional. Esto, desde luego, hace susceptible al

\footnotetext{
${ }^{25}$ Es común encontrar en la crítica anticosmopolita la consideración de este proyecto como un paradigma homogéneo, cuya naturaleza se inscribe en la instauración de un Estado de Estados, civitas maxima o Estado mundial, implicando un proceso de homogeneización política, económica, jurídica y cultural que finalmente conforma la política global del imperialismo occidental. No negamos la pertinencia teórica y práctica de tales críticas, en razón del desarrollo histórico del sistema internacional y su actual situación. No obstante, el proyecto cosmopolita contemporáneo dista de suscribir tal visión, constituyéndose a su vez como un paradigma crítico a tales tesis. En este sentido, y como ocurre con las más destacadas corrientes de pensamiento, es fácil elaborar caricaturas y esquematizaciones sobre-simplificadoras que conducen a una comprensión incorrecta del mismo.
} 
cosmopolitismo de la crítica de las intervenciones humanitarias y de la doctrina de la Guerra Justa. $^{26}$

\section{Conclusiones}

El proyecto cosmopolita contemporáneo comprende posturas fuertes y débiles, en un amplio espectro graduado, pudiendo sostener, sobre fundamentos kantianos, tanto la propuesta de un Estado mundial como de una Federación de Estados. Es en la visión de los teóricos neokantianos que proponen la tesis de un sistema global federal descentralizado donde encontramos un acento central en la idea de una ciudadanía mundial, inscrita en el marco de una sociedad civil global y una opinión pública global, categorías que en el ámbito de las tesis más fuertes del Estado mundial tienden a desdibujarse. Es en la visión federalista (b) donde la discusión y decisión sobre los conflictos bélicos y el uso de la fuerza a nivel internacional pueden verse influidos en mayor medida por una sociedad civil y una opinión pública globales, determinando que la naturaleza del sistema internacional tenga un carácter más horizontal, efectuada a través de organizaciones civiles, instituciones y redes formales e informales. ${ }^{27}$ Ello se constituye como una instancia crítica frente a los

\footnotetext{
${ }^{26}$ El problema de las intervenciones humanitarias en el cosmopolitismo contemporáneo es sumamente complejo e incluye una gama amplia de posturas. Kant estableció en los artículos preliminares hacia la paz perpetua un principio de no intervención, no obstante, como Santiago (2004: 134) señala, mantener una postura rígida de este principio tendría consecuencias moralmente indeseables, aunque: "no hay una manera sencilla de resolver el problema surgido del principio de no intervención". Para un estudio sobre este tema, véase La guerra humanitaria. Pasado y presente de una controversia filosófica (2013), donde Santiago examina sistemáticamente el concepto de guerra humanitaria, en el contexto del nuevo humanitarismo y las intervenciones humanitarias, basados en la doctrina de los derechos humanos, aunque sin pretender defender ni atacar el intervencionismo militar con fines humanitarios, sino comprender el fenómeno de su revitalización actual y consecuente debate filosófico. Para un argumento a favor de la justificación moral de la intervención, véase Archibugi, 2003: 147; Tesón, 2003: 93 (así mismo, para un análisis de estas posturas, véase Santiago, Ibíd.: 126).

${ }^{27}$ El cosmopolitismo contemporáneo reconoce la existencia de un plexo de actores globales, superpuestos en distintos niveles institucionales e informales, que desempeñan un papel importante en el contexto de un sistema global democrático. Estos actores son instituciones $\mathrm{y}$ organismos estatales, regionales $\mathrm{e}$ internacionales, asociaciones civiles, organizaciones no gubernamentales, así como empresas trasnacionales. Es en este plexo donde se comprende la participación de una naciente sociedad civil global, cuyo actor fundamental son los ciudadanos del mundo. En todo caso, el cosmopolitismo contemporáneo comprende que tanto la sociedad civil global como la opinión pública global se encuentran in status nascendi, por lo que se
} 
procesos políticos globales verticales, dominados por actores estatales, empresariales o por burocracias especializadas, al margen del control democrático de la ciudadanía.

Finalmente, el concepto de ciudadanía mundial constituye el núcleo teórico y político del cosmopolitismo contemporáneo. La idea de una ciudadanía mundial tiene un interés no sólo filosófico, conceptual o académico, sino que posee una importancia fundamental en el debate político en los días presentes sobre la perspectiva de una reforma a las instituciones globales en sentido democrático, que enfrente los graves problemas derivados del proceso de globalización. ${ }^{28}$ La característica esencial del cosmopolitismo político contemporáneo es la participación de la ciudadanía mundial en los ámbitos de una esfera pública global y una opinión pública global. Tal participación posibilita que la naturaleza del proceso de democratización del sistema internacional tenga un carácter más horizontal, efectuada a través de organizaciones civiles, instituciones y redes formales e informales. Ello se constituye como una instancia crítica frente a los procesos políticos globales verticales, dominados por actores estatales, empresariales o por burocracias especializadas, al margen del control democrático de la ciudadanía. La existencia de una sociedad civil global y una opinión pública global tienen mayores posibilidades de desarrollo en razón de las nuevas tecnologías de la información y la comunicación

acentúa la existencia de redes informales de organización y participación de carácter global, complementadas con aquellas de alcance regional y nacional.

${ }^{28}$ La perspectiva de una ciudadanía mundial se encuentra latente en el debate público, por ejemplo, en el año 2012 fue dado a conocer el Manifesto for a Global Democracy, firmado por intelectuales como Noam Chomsky y David Held. El Manifiesto expresa que las profundas transformaciones tecnológicas y económicas propias de la globalización no han sido acompañadas por una evolución similar de las instituciones públicas encargadas de su regulación, por lo que: "la economía se ha globalizado, pero las instituciones políticas y la democracia, no". El Manifiesto hace un llamado al avance de nuevas formas de gobernanza global (global governance) especializadas en el desarme, el crecimiento estable, equitativo y sostenible, así como la protección del medio ambiente. Ello implica una reforma en sentido democrático de las instituciones de gobernanza global, acompañada por formas más extensas y profundas de democracia, cuyo objetivo es la institucionalización participativa de una democracia global. Ahora bien, la necesidad de una reforma democrática del sistema global, así como la participación de la ciudadanía mundial en este proceso, ha sido señalada no solamente por los movimientos sociales y los estudiosos de la globalización, sino también a nivel institucional. Un ejemplo importante lo encontramos en el Informe sobre Desarrollo Humano 2013, elaborado por el Programa de las Naciones Unidas para el Desarrollo (PNUD). El Informe pondera la importancia de la sociedad civil mundial, única capaz de actuar como una fuerza compensatoria para los Estados y los mercados, así como para presionar la rendición de cuentas de las instituciones de gobernanza global (PNUD, 2013: 110). 
características del proceso de globalización, son estos medios los que posibilitan la información, comunicación e interacción creciente sobre problemas de alcance global. En todo caso, se comprende que tanto la sociedad civil global como la opinión pública global se encuentran in status nascendi, por lo que se acentúa la existencia de redes informales de organización y participación de carácter global, complementadas con aquellas de alcance regional y nacional.

Así mismo, El ciudadano del mundo es el sujeto central del derecho cosmopolita, cuyo núcleo normativo y jurídico está conformado por la doctrina de los derechos humanos. La evolución del derecho internacional ha determinado que los individuos, y no los Estados, sean los sujetos primordiales del mismo. Son los derechos humanos aquellos que constituyen aquel mínimo que posibilita la constitución de la persona como tal, así como para su actuación plena en los procesos democráticos como ciudadano de un determinado Estado o región y como ciudadano del mundo. Es importante señalar que tales derechos no deben entenderse solamente como derechos negativos o mínimos, sino como derechos sociales o positivos en sentido amplio. No resulta adecuado concebir el núcleo del derecho cosmopolita en sentido restrictivo, como meros derechos negativos o como un mero derecho de hospitalidad, entendido como un derecho de visita (Besuchsrecht) y no un derecho de huésped (Gastrecht), de acuerdo con el derecho natural, tal como Kant lo expone en Zum ewigen Frieden (AA, VIII 358). Una ampliación hacia los derechos positivos o sociales corresponde a la historia del progresivo ensanchamiento de la esfera de los derechos durante el siglo XX: del derecho de huelga y los derechos sociales, al derecho a la educación, salud y trabajo, hasta los nuevos derechos a la paz, al medio ambiente sano y a la información. La idea de la inclusión de estos derechos sociales y económicos es absolutamente central en la visión del enfoque de las capacidades de Martha Nussbaum, el enfoque de la justicia global de Thomas Pogge, el enfoque garantista de Luigi Ferrajoli, así como en la teoría jurídica de los derechos fundamentales de Robert Alexy, entendidos 
como derechos a acciones positivas del Estado, como prestaciones en sentido amplio. ${ }^{29} \mathrm{En}$ una situación de injusticia social extrema como la que se vive en distintas latitudes del mundo, imposibilita que los individuos puedan constituirse ya no se diga como ciudadanos mundiales, sino que puedan ejercer un mínimo de acción y participación en lo público aún en lo local. Es por ello por lo que la filosofía cosmopolita contemporánea elabora una crítica profunda al estado actual de fuerte desigualdad y pobreza global, una crítica y aspiración que debemos suscribir completamente.

\section{Bibliografía}

Alexy, Robert (1993): Teoría de los derechos fundamentales. Madrid: Centro de Estudios Constitucionales.

- (1994): El concepto y la validez del derecho. Barcelona: Gedisa.

Bobbio, Norberto (2001): "Democracia y sistema internacional", en El futuro de la democracia.

Carson, Thomas (1998): 'Perpetual Peace: What Kant Should Have Said". Social Theory and Practice, No. 14 pp. 173-214.

Ferrajoli, Luigi (2001): Los fundamentos de los derechos fundamentales. Madrid, Trotta.

- (2004a): Derechos y garantías. La ley del más débil. Madrid, Trotta.

- (2004b): Razones jurídicas del pacifismo. Madrid: Trotta.

Habermas, Jürgen (1993): Faktizität und Geltung. Beiträge zur Diskurstheorie des Rechts und des demokratischen Rechtsstaats. Frankfurt am Main: Suhrkamp Verlag.

\footnotetext{
${ }^{29}$ Alexy (1993: 495; 1994: 154) argumenta que entre los derechos humanos básicos se encuentran probablemente derechos sociales fundamentales mínimos, como el derecho a un mínimo vital. Ahora bien, Alexy (1993: 419) comenta que la cuestión de si y en qué medida a las disposiciones de derechos fundamentales deben adscribirse normas que confieren derechos sociales o a prestaciones en sentido amplio es una de las más discutidas en la dogmática actual de los derechos fundamentales. Finalmente, los derechos fundamentales sociales, considerados como derechos a prestaciones, afirma Alexy, comprenden dos dimensiones: prestaciones fácticas y prestaciones normativas. A su vez, se dividen en: a) derechos de protección; b) derechos a organización y procedimiento y; c) derechos a prestaciones en sentido estricto.
} 
- (1995): Kants Idee des Ewigen Friedens aus dem historischen Abstand von 200 Jahren, Kritische, Justiz, 28, pp. 293-319.

- (1988): Ensayos políticos. Barcelona: Península.

- (1999): La inclusión del otro. Estudios de teoría política. Barcelona: Paidós.

- (2000): La constelación posnacional. Ensayos políticos. Barcelona, Paidós.

- (2014): "A political constitution for the pluralist world society". Journal of Chinese Philosophy, vol. 34, Issue 3, pp. 331-343.

- (2008a): "The constitutionalization of International Law and the Legitimation Problems of a Constitution of World Society“, Constellations, Vol. 15, Issue 4, pp.444-455.

- (2008b): El derecho internacional en la transición hacia un escenario posnacional. Barcelona: Katz.

Held, David (1997): La democracia y el orden global. Del Estado moderno al gobierno cosmopolita. Barcelona: Paidós.

Hodgson, Louis-Philippe (2010): "Kant on the Right to Freedom: A defense“. Ethics, No. 120, pp. 791-819.

- (2012): "Realizing external freedom: the Kantian argument for a world state“, en Ellis, Elisabeth

(ed.), Kant's Political Theory: Interpretations and Applications. PA: Pennsylvania State University Press, pp. 101-134.

Höffe, Otfried (1999). Demokratie im Zeitalter der Globalisierung. München, C.H. Beck.

- (2000): Derecho intercultural. Barcelona: Gedisa.

- (2006): Kant's Cosmopolitan Theory $f$ Law and Peace. New York: Cambridge University Press.

Kaldor, Mary Kaldor, Mary (2003): "The idea of global civil society". International Affairs, no. 79, 3, pp. 583-593.

- (2005): La sociedad civil global. Una respuesta a la guerra. Barcelona: Tusquets. 


\section{ARTÍCULOS}

Kant, Immanuel: Idee zu einer allgemeinen Geschichte in weltbürgerlicher Absicht (1784), en Kants Werke. Akademieausgabe. Band VIII. Abhandlungen nach 1781. Das Bonner Kant-Korpus, Elektronische Edition der Gesammelten Werke Immanuel Kants, pp. 15-31. (Sigla: Idee, AA VIII).

-: Mutmaßlicher Anfang der Menschengeschichte (1786), en Kants Werke. Akademieausgabe. Band VIII. Abhandlungen nach 1781. Das Bonner Kant-Korpus, Elektronische Edition der Gesammelten Werke Immanuel Kants, pp. 107-123. (Sigla: MA, AA VIII). 